\title{
ON DIFFERENT DEFINITIONS OF STRAIN TENSORS IN GENERAL SHELL THEORIES OF VEKUA-AMOSOV TYPE
}

\author{
Sergey I. Zhavoronok \\ Institute of Applied Mechanics of Russian Academy of Sciences, Moscow, RUSSIA
}

\begin{abstract}
Several possible definitions of strains in a general shell theory of I.N. Vekua -A.A. Amosov type are considered. The higher-order shell model is defined on a two-dimensional manifold within a set of field variables of the first kind determined by the expansion factors of the spatial vector field of the translation. Two base vector systems are introduced, the first one so-called concomitant corresponds to the cotangent fibration of the modelling surface while the other is defined on a surface equidistant to the modelling one. The distortion appears as a two-point tensor referred to both base systems after covariant differentiation of the translation vector field. Thus, two main definitions of the strain tensor become possible, the first one referred to the main basis whereas the second to the concomitant one. Some possible simplifications of these tensors are considered, and the interrelation between the general theory of A.A. Amosov type and the classical ones is shown.
\end{abstract}

Keywords: hierarchical modeling of shells, dimensional reduction, analytical continuum dynamics, strain tensors, stress tensors

\section{О РАЗЛИЧНЫХ ОПРЕДЕЛЕНИЯХ ТЕНЗОРОВ ДЕФОРМАЦИИ В ОБЩЕЙ ТЕОРИИ ОБОЛОЧЕК И.Н. ВЕКУА - А.А. АМОСОВА}

\author{
С.И. Жаворонок \\ Институт прикладной механики Российской Академии наук, Москва, Россия
}

\begin{abstract}
Аннотация: Рассмотрены различные возможные определения деформации в общей теории оболочек И.Н. Векуа А.А. Амосова. Модель оболочки высшего порядка определена на двумерном многообразии множеством переменных поля первого рода, определенных коэффициентами разложения пространственного поля вектора перемещения по некоторой системе функций. Введены две системы базисных векторов, а именно сопутствующий базис в касательном расслоении многообразия, соответствующего реперной поверхности оболочки, и основной базис, соответствующей произвольной поверхности, эквидистантной реперной. Введены также двухточечные представления тензора дисторсии в тензорном произведении касательных пространств к реперной и эквидистантной поверхностям, порождаемые градиентом поля вектора перемещения. Таким образом возникают два определения тензора деформации оболочки как трехмерного тела, первый из них отнесен к основному базису, тогда как второй - к сопутствующему. Рассмотрены некоторые упрощенные формы записи тензоров деформации, соответствующие теориям низших порядков, и указано соответствие между теорией оболочек в форме А.А. Амосова и классическими теориями.
\end{abstract}

Ключевые слова: иерархические модели оболочек, редукция пространственной размерности, аналитическая механика континуальных систем, тензоры деформации, тензоры напряжения

\section{INTRODUCTION}

The intrinsic kinematics of the classical shell theory is based on two parameters, the tangent and bending strains of the modelling surface of a shell $[1,2]$. These quantities were initially defined using the orthogonal coordinates referred to principal curvature lines of the modelling surface [3] while the modern shell theory is based on the tensor analysis [4]. Various refined theories of shells use different strain definitions $[5,6]$ referred to the orthogonal coordinates or tensor-based definitions [7-10]. One should note that most of cited works introduce different strain tensor definitions; several theories 
operates with tensor coefficients with no description of base systems in details $[9,10]$. Indeed, at least two base vector triads could be used in generalized shell theories [7, 10-12], the spatial (or main) basis of an arbitrary curvilinear frame normally attached to the modelling surface and the concomitant one [7, $11,12]$; the first one generates a set of space tensors whereas the second one generates surface tensors [7]. Let us note that more complex models use twopoint tensor definitions [13-15]. Such a situation leads to different work-conjugated pairs of stress and strain tensors and different formulation of variational principles [16] for a shell theory.

This work is a brief systematization of strain and stress tensor representations in higher-order shell theories. The two-point spatial distortion tensor [15] is used as a background; two main formulations for the spatial strain and four for the stress tensors allowing one to obtain the surface tensors after integration over the thickness are introduced. It is shown that a clear analogy of these quantities and the tensors used in the nonlinear solid mechanics appears if a surface equidistant to the base one is obtained as a result of the imagined motion along normal, and the spatial, or main, basis could be interpreted as "acting" one while the concomitant basis as "initial" one [18]. The appropriate workconjugated tensor pairs are defined, the strain energy is formulated, and the sets of surface stress and strain tensors defining a higher-order shell theory are described. Finally, the interrelation between the strain tensors in the higher-order theory [15] and the classical shell theories is shown.

\section{GEOMETRY OF THE MODELLING SURFACE OF A SHELL}

Let us consider a two-dimensional manifold:

$$
\begin{gathered}
S \subset l^{3}: \quad \forall M \in S \quad \mathbf{r}(M)=x^{i}(M) \mathbf{e}_{i} ; \\
\exists \xi^{\alpha}: \quad S \rightarrow D_{\xi} \subseteq l^{2}, \quad i=\overline{1,3}, \alpha=\overline{1,2} ; \\
x^{i}\left(\xi^{\alpha}\right) \in C^{(2)}\left(D_{\xi}\right) \Rightarrow \exists d \mathbf{r}(M)=\mathbf{r}_{\alpha} d \xi^{\alpha} \\
\mathbf{r}_{\alpha}=\left(\partial x^{i} / \partial \xi^{\alpha}\right) \mathbf{e}_{i}, \quad \operatorname{Rk}\left(\partial x^{i} / \partial \xi^{\alpha}\right)=2 ;
\end{gathered}
$$

$\xi^{\alpha}$ are curvilinear coordinates on $S$. The tangent fibration $T S$ can be defined by:

$$
T S=\coprod_{M \in S} T_{M} S, \quad T_{M} S=\left\{y^{\alpha} \mathbf{r}_{\alpha} \mid y^{\alpha} \in \square\right\} .
$$

The fibration $T S$ is furnished by the metric a

$$
\begin{gathered}
\mathbf{a}: \quad T S \times T S \rightarrow \square_{+}, \quad \mathbf{a}=a_{\alpha \beta} \mathbf{r}^{\alpha} \mathbf{r}^{\beta}, \\
a_{\alpha \beta}=\mathbf{r}_{\alpha} \cdot \mathbf{r}_{\beta}, \quad a=\operatorname{det}\left(a_{\alpha \beta}\right), \quad a \neq 0 \Rightarrow \\
\forall M \in S \quad \exists a^{\gamma \beta}, \quad a^{\gamma \beta} a_{\alpha \beta}=\delta_{\alpha}^{\gamma}, \quad \mathbf{r}^{\gamma}=a^{\gamma \beta} \mathbf{r}_{\beta},
\end{gathered}
$$

and the discriminant tensor $\mathbf{T}=\mathrm{T}^{\alpha \beta} \mathbf{r}_{\alpha} \mathbf{r}_{\beta}$. The cotangent fibration $T^{\mathrm{e}} S$ is given as

$$
T^{\mathrm{e}} S=\coprod_{M \in S} T_{M}^{\mathrm{e}} S, \quad T_{M}^{\mathrm{e}} S=\left\{y_{\alpha} \mathbf{r}^{\alpha} \mid y_{\alpha} \in \square\right\} .
$$

The orientation of the manifold $S$ is defined by the normal unit vector $\mathbf{n}$ :

$$
\mathbf{n}(M)=a^{-1 / 2} \mathbf{r}^{1} \wedge \mathbf{r}^{2}=\mathrm{T}^{\alpha \beta} \mathbf{r}_{\alpha} \wedge \mathbf{r}_{\beta},
$$

so that we obtain the normal fibration of $S$ :

$$
N S=\coprod_{M \in S} N_{M} S, \quad N_{M} S=\{y \mathbf{n} \mid y \in \square\} .
$$

The differentiation of $\mathbf{r}_{\alpha}, \mathbf{n}$ is defined by

$$
\begin{gathered}
\partial_{\beta} \mathbf{r}_{\alpha}=\Gamma_{\alpha \beta}^{\gamma} \mathbf{r}^{\gamma}+b_{\alpha \beta} \mathbf{n}, \quad \partial_{\beta} \mathbf{r}^{\gamma}=-\Gamma_{\alpha \beta}^{\gamma} \mathbf{r}^{\beta}+b_{\beta}^{\cdot \gamma} \mathbf{n}, \\
\partial_{\beta} \mathbf{n}=-b_{\alpha \beta} \mathbf{r}^{\alpha} .
\end{gathered}
$$

\section{KINEMATICS OF A DEFORMING MODELLING SURFACE OF A SHELL}

Let us define a vector field $\mathbf{u}_{s}$ :

$$
\begin{gathered}
\mathbf{u}_{S}: S \times(\square+\cup\{0\}) \rightarrow T^{\mathrm{e}} S \oplus N S, \\
\mathbf{u}_{S}=\bar{u}_{\alpha}\left(\xi^{\beta}, t\right) \mathbf{r}^{\alpha}+w\left(\xi^{\beta}, t\right) \mathbf{n} ;
\end{gathered}
$$

here $\bar{u}_{\alpha}$ correspond to the tangent translation while $w$ is the deflection of a surface $S$. Introducing Nabla operator $\bar{\nabla}=\mathbf{r}^{\beta} \partial_{\beta}$ we have 
On Different Definitions of Strain Tensors in General Shell Theories of Vekua-Amosov Type

$$
\begin{gathered}
\overline{\mathbf{d}} \equiv \bar{\nabla} \otimes \mathbf{u}_{S}=\overline{\bar{d}}_{\beta \alpha} \mathbf{r}^{\beta} \mathbf{r}^{\alpha}+\vartheta_{\beta} \mathbf{r}^{\beta} \mathbf{n}, \\
\overline{\bar{d}}_{\beta \alpha}=\nabla_{\beta} \bar{u}_{\alpha}-b_{\alpha \beta} w, \quad \vartheta_{\beta}=\partial_{\beta} w+b_{\beta}^{\cdot \alpha} \bar{u}_{\alpha} .
\end{gathered}
$$

Let us consider the deformed surface $S^{\prime}$ :

$$
\forall M \in S \quad \mathbf{r}^{\prime}(M, t)=\mathbf{r}(M)+\mathbf{u}_{S}(M, t)
$$

Thus, we obtain the tangent fibration $T S^{\prime}$ and normal one $N S^{\prime}$ for the deformed surface $S^{\prime}$ :

$$
\begin{gathered}
T S^{\prime}=\coprod_{M \in S}\left\{y^{\alpha} \mathbf{r}_{\alpha}^{\prime}(M) \mid y^{\alpha} \in \square\right\}, \\
\mathbf{r}_{\alpha}^{\prime}(M)=\mathbf{r}_{\alpha}(M)+\bar{d}_{\alpha \cdot \beta}^{\beta} \mathbf{r}_{\beta}+\vartheta_{\alpha} \mathbf{n} ; \\
\mathbf{a}^{\prime}=a_{\alpha \beta}^{\prime} \mathbf{r}^{\alpha} \otimes \mathbf{r}^{\beta}=\left(a_{\alpha \beta}+2 \varepsilon_{\alpha \beta}\right) \mathbf{r}^{\alpha} \mathbf{r}^{\beta}, \\
\varepsilon_{\alpha \beta} \approx \frac{1}{2}\left(\nabla_{\alpha} \bar{u}_{\beta}+\nabla_{\beta} \bar{u}_{\alpha}\right)-b_{\alpha \beta} w ; \\
N S^{\prime}=\coprod_{M \in S}\left\{y \mathbf{n}^{\prime} \mid y \in \square\right\}, \\
\mathbf{n}^{\prime}=\sqrt{a^{\prime} / a} \mathrm{~T}^{\alpha \beta} \mathbf{r}_{\alpha}^{\prime} \mathbf{r}_{\beta}^{\prime} \approx \mathbf{n}-\vartheta_{\alpha} \mathbf{r}^{\alpha} .
\end{gathered}
$$

Here $\varepsilon_{\alpha \beta}$ is the surface tangent strain while its bending strain is defined by the tensor $\mathbf{u}$ :

$$
\begin{gathered}
\mathbf{b}^{\prime}=\left(b_{\alpha \beta}+2 щ_{\alpha \beta}\right) \mathbf{r}^{\alpha} \mathbf{r}^{\beta}, \quad щ_{\alpha \beta}= \\
=\frac{1}{2}\left(\nabla_{\alpha} \vartheta_{\beta}+\nabla_{\beta} \vartheta_{\alpha}\right)+\frac{1}{2}\left(b_{\alpha}^{\cdot \gamma} \bar{d}_{\beta \gamma}+b_{\beta}^{\cdot \gamma} \bar{d}_{\alpha \gamma}\right),
\end{gathered}
$$

here the second-order terms are neglected as provided by linear shell theories [2].

\section{GEOMETRY OFTHE SHELLAS A THREE- DIMENSIONAL BODY}

Let us consider hence the shell as $V \in \square^{3}[15]$ :

$$
\begin{gathered}
\forall M_{\zeta} \in V \quad \exists \mathbf{R}\left(M_{\zeta}\right)=\mathbf{r}(M)+\zeta h \mathbf{n}(M), \\
\zeta \in D_{\zeta}=[-1,1]
\end{gathered}
$$

here $2 h$ denotes the shell thickness. We can define hence an equidistant surface $S_{\zeta}$ for any $\zeta \neq 0$, e.g. a manifold with the fibrations

$$
T S_{\zeta}=\coprod_{M \in S}\left\{y^{\alpha} \mathbf{R}_{\alpha} \mid y^{\alpha} \in \square\right\},
$$

$$
\begin{gathered}
\mathbf{R}_{\alpha}=\partial_{\alpha} \mathbf{R}=A_{\alpha \cdot \beta}^{\beta} \mathbf{r}_{\beta}, \quad A_{\alpha \cdot}^{\beta}=\delta_{\alpha}^{\beta}-\zeta b_{\alpha \cdot}^{\beta} ; \\
N S_{\zeta}=\coprod_{M \in S}\{y \mathbf{n} \mid y \in \square\} \equiv N S: \\
T V=\coprod_{M \in S}\left\{y^{\alpha} \mathbf{R}_{\alpha}+y \mathbf{n}\right\}=T S_{\zeta} \oplus N S .
\end{gathered}
$$

here the linear transform given by $A_{\alpha \cdot}^{\cdot \beta}[7,15]$ defines the parallel shifting. This transform is nonsingular if $A=\operatorname{det}\left(A_{\alpha^{*}}^{\beta}\right) \neq 0$, i. e. if [15]

$$
\begin{gathered}
A=1-2 \zeta h H+\zeta^{2} h^{2} K=\left(1-\zeta h k_{1}\right)\left(1-\zeta h k_{2}\right) \neq 0 \\
\Leftrightarrow h<\inf _{M E S} k_{\alpha}^{-1}, \quad \alpha=1,2 ;
\end{gathered}
$$

where $k_{1,2}$ are principal curvatures of $S$. Accounting for, we could obtain $[7,14]$

$$
\begin{gathered}
\mathbf{r}_{\beta}=A_{\cdot \beta}^{\alpha \cdot} \mathbf{R}_{\alpha}, \quad A_{\cdot \gamma}^{\alpha \cdot} A_{\beta}^{\cdot{ }^{\gamma}}=\delta_{\beta}^{\alpha} ; \\
A_{\cdot \beta}^{\alpha \cdot}=A^{-1}\left(\delta_{\beta}^{\alpha}-\zeta h \mathbf{T}^{\alpha \lambda} \mathrm{T}_{\beta \mu} b_{\lambda \cdot}^{\mu}\right) .
\end{gathered}
$$

The metric $\mathbf{g}$ on $T S$ is defined by (1.18) [7]:

$$
\begin{gathered}
g_{\alpha \beta}=A_{\alpha \cdot}^{\cdot \gamma} \cdot A_{\beta}^{\cdot \delta} a_{\gamma \delta}=a_{\alpha \beta}-2 \zeta h b_{\alpha \beta}+\zeta^{2} h^{2} c_{\alpha \beta}, \\
g=\operatorname{det}\left(g_{\alpha \beta}\right)=\operatorname{det}\left(A_{\alpha \cdot}^{\cdot \beta}\right)^{2} a=A^{2} a, \\
c_{\alpha \beta}=b_{\alpha \cdot} \cdot b_{\beta \gamma} . \quad H=\frac{1}{2} b_{\alpha \cdot}^{\cdot \alpha}, \quad K=\operatorname{det}\left(b_{\alpha \cdot}^{\cdot \beta}\right) .
\end{gathered}
$$

For more details see $[14,15,17]$.

\section{PARALLEL SHIFTING TENSORS}

One could have two interpretations of parallel shift tensors. Let us consider a unit tensor I:

$$
\begin{gathered}
\mathbf{I}=\mathbf{r}_{\alpha} \mathbf{r}^{\alpha}=A_{\cdot \alpha}^{\beta \cdot} \mathbf{R}_{\beta} \mathbf{r}^{\alpha}=A_{\gamma}^{\cdot \alpha} \mathbf{r}_{\alpha} \mathbf{R}^{\gamma}= \\
=A_{\cdot \alpha}^{\beta \cdot} A_{\gamma}^{\alpha \alpha} \mathbf{R}_{\beta} \mathbf{R}^{\gamma}=\delta_{\gamma}^{\beta} \mathbf{R}_{\beta} \mathbf{R}^{\gamma}=\mathbf{R}_{\beta} \mathbf{R}^{\beta} ;
\end{gathered}
$$


such an interpretation was introduced by I.N. Vekua in [7]; the left indices of $A_{\alpha}^{\cdot \beta}$ must be raised and lowered by the metric tensor $\mathbf{g}$ whereas the right ones - by the metric a [7]:

$$
\begin{aligned}
& \mathbf{I}=A_{\cdot \alpha}^{\beta \cdot} \mathbf{R}_{\beta} \mathbf{r}^{\alpha}=A_{\cdot \alpha}^{\beta \cdot} g_{\beta \gamma} \mathbf{R}^{\gamma} \mathbf{r}^{\alpha}=A_{\gamma \alpha} \mathbf{R}^{\gamma} \mathbf{r}^{\alpha}, \\
& \mathbf{I}=A_{\cdot \alpha}^{\beta \cdot} \mathbf{R}_{\beta} \mathbf{r}^{\alpha}=A_{\cdot \alpha}^{\beta \cdot} a^{\alpha \delta} \mathbf{R}_{\beta} \mathbf{r}_{\delta}=A^{\beta \delta} \mathbf{R}_{\beta} \mathbf{r}_{\delta}, \ldots
\end{aligned}
$$

The Vekua unit tensors (1.19) allow to define vector fields on the fibrations $T^{e} S, T_{\zeta}{ }^{e} S$ :

$$
\begin{gathered}
\mathbf{u}_{T}: V \times\left(\square_{+} \cup\{0\}\right) \rightarrow T^{\mathrm{e}} S_{\zeta}, \mathbf{u}_{T}=\hat{u}_{\alpha} \mathbf{R}^{\alpha} ; \\
\mathbf{u}_{T}: V \times\left(\square_{+} \cup\{0\}\right) \rightarrow T^{\mathrm{c}} S, \mathbf{u}_{T}=u_{\alpha} \mathbf{r}^{\alpha} ; \\
\mathbf{u}_{T} \cdot \mathbf{I}=A_{\cdot \alpha}^{\beta \cdot} \hat{u}_{\gamma} \mathbf{R}^{\gamma} \cdot \mathbf{R}_{\beta} \mathbf{r}^{\alpha}=A_{\cdot \alpha}^{\gamma} \hat{u}_{\gamma} \mathbf{r}^{\alpha}=u_{\alpha} \mathbf{r}^{\alpha} ; \\
\mathbf{I} \cdot \mathbf{u}_{T}=A_{\gamma \cdot}^{\alpha} u_{\beta} \mathbf{r}^{\beta} \cdot \mathbf{r}_{\alpha} \mathbf{R}^{\gamma}=A_{\gamma \cdot}^{\beta} u_{\beta} \mathbf{R}^{\gamma}=\hat{u}_{\gamma} \mathbf{R}^{\gamma} ; \\
\hat{u}_{\gamma}=A_{\gamma}^{\beta} u_{\beta}, \quad u_{\alpha}=A_{\cdot \alpha}^{\beta \cdot} \hat{u}_{\beta} .
\end{gathered}
$$

On the other hand we have the tensor $\mathbf{A}(\zeta)$ :

$$
\begin{aligned}
& \mathbf{A}=\mathbf{R}_{\alpha} \mathbf{r}^{\alpha}=A_{\alpha \cdot}^{\cdot \beta} \mathbf{r}_{\beta} \mathbf{r}^{\alpha}=A_{\alpha \cdot}^{\cdot \beta} \mathbf{R}_{\beta} \mathbf{R}^{\alpha} ; \\
& \mathbf{A}^{-1}=\mathbf{r}_{\alpha} \mathbf{R}^{\alpha}=A_{\cdot \alpha}^{\beta \cdot} \mathbf{R}_{\beta} \mathbf{R}^{\alpha}=A_{\alpha}^{\cdot \beta} \mathbf{r}_{\beta} \mathbf{r}^{\alpha} .
\end{aligned}
$$

Let us note the analogy of $\mathbf{A}$ and the position gradients [18] if the vector $\mathbf{r}_{\alpha}$ is interpreted like "initial basis" and $\mathbf{R}_{\alpha}$ like "acting basis":

$$
\mathbf{A} \cdot \mathbf{r}_{\beta}=\mathbf{R}_{\beta}, \quad \mathbf{A}^{-1} \cdot \mathbf{R}_{\alpha}=\mathbf{r}_{\alpha}, \quad \cdots
$$

This tensor provides the linear morphism

$$
\begin{gathered}
\mathbf{A}: T S \rightarrow T S_{\zeta}, \mathbf{A}: T^{\mathrm{e}} S_{\zeta} \rightarrow T^{\mathrm{e}} S ; \\
\mathbf{A}^{-1}: T^{\mathrm{c}} S \rightarrow T^{\mathrm{c}} S_{\zeta}, \mathbf{A}^{-1}: T S_{\zeta} \rightarrow T S .
\end{gathered}
$$

\section{SPATIAL KINEMATICS OF A SHELL}

Let $\mathbf{u}(M, \zeta, t)$ be translation vector field:

$$
\begin{gathered}
\mathbf{u}: V \times\left(\square_{+} \cup\{0\}\right) \rightarrow T^{\mathrm{e}} V, \\
\mathbf{u}=u_{\beta} \mathbf{R}^{\beta}+u_{3} \mathbf{n} .
\end{gathered}
$$

Since the fibration $T V$ is defined by (1.15) and the morphism (1.23) of bundles exists, $T S_{\zeta}=T S \times D_{\zeta}$ we could represent $T^{e} V$ as

$$
T^{\mathrm{c}} V=T^{\mathrm{c}} S_{\zeta} \oplus N S \square T^{\mathrm{c}} S \oplus N S \times D_{\zeta},
$$

thus, the vector field $\mathbf{u}$ can be determined as

$$
\begin{gathered}
\mathbf{u}: S \times D_{\zeta} \times(\square+\{0\}) \rightarrow T^{\mathrm{c}} S \oplus N S \times D_{\zeta} \\
\Rightarrow \quad \mathbf{u}=u_{\beta} \mathbf{r}^{\beta}+u_{3} \mathbf{n},
\end{gathered}
$$

where the basis $\mathbf{r}^{\alpha}, \mathbf{n}$ does not depend on $\zeta$. Let $\nabla=\mathbf{R}^{\alpha} \partial_{\alpha}+\mathbf{n} \partial_{\zeta}$ be Nabla operator on $T V=T S_{\zeta} \oplus N S$; thus, we obtain the two-point formulation of the distortion $\mathbf{d}=\nabla \otimes \mathbf{u}[14]$ :

$$
\begin{gathered}
\mathbf{d}=\left(\mathbf{R}^{\alpha} \partial_{\alpha}+\mathbf{n} \partial_{\zeta}\right) \otimes\left(u_{\beta} \mathbf{r}^{\beta}+u_{3} \mathbf{n}\right)= \\
=\bar{d}_{\alpha \beta} \mathbf{R}^{\alpha} \mathbf{r}^{\beta}+\bar{d}_{\alpha 3} \mathbf{R}^{\alpha} \mathbf{n}+\bar{d}_{3 \beta} \mathbf{n} \mathbf{r}^{\beta}+\bar{d}_{33} \mathbf{n n} ; \\
\bar{d}_{\alpha \beta}=\nabla_{\alpha} u_{\beta}-b_{\alpha \beta} u_{3}, \quad \bar{d}_{\alpha 3}=\nabla_{\alpha} u_{3}+b_{\alpha \cdot}^{\cdot \beta} u_{\beta} ; \\
\bar{d}_{3 \beta}=\partial_{\zeta} u_{\beta}, \quad \bar{d}_{33}=\partial_{\zeta} u_{3} .
\end{gathered}
$$

Taking into account (1.20) we have [15]

$$
\begin{gathered}
\mathbf{d}=d_{\delta \beta} \mathbf{r}^{\delta} \mathbf{r}^{\beta}+d_{\delta 3} \mathbf{r}^{\delta} \mathbf{n}+d_{3 \beta} \mathbf{n} \mathbf{r}^{\beta}+d_{33} \mathbf{n n}= \\
=\hat{d}_{\alpha \gamma} \mathbf{R}^{\alpha} \mathbf{R}^{\gamma}+\hat{d}_{\alpha 3} \mathbf{R}^{\alpha} \mathbf{n}+\hat{d}_{3 \gamma} \mathbf{n} \mathbf{R}^{\gamma}+\hat{d}_{33} \mathbf{n n} ; \\
\hat{d}_{\alpha \gamma}=A_{\gamma \cdot}^{\beta} \bar{d}_{\alpha \beta}, \quad \hat{d}_{\alpha 3}=\bar{d}_{\alpha 3} ; \\
\hat{d}_{3 \gamma}=A_{\gamma \cdot}^{\beta} \bar{d}_{3 \beta}, \quad \hat{d}_{33}=\bar{d}_{33} ; \\
d_{\delta \beta}=A_{\delta \delta}^{\alpha \cdot} \bar{d}_{\alpha \beta}, \quad d_{\delta 3}=A_{\cdot \delta}^{\alpha \cdot} \bar{d}_{\alpha 3} ; \\
d_{3 \beta}=\bar{d}_{3 \beta}, \quad d_{33}=\bar{d}_{33} .
\end{gathered}
$$

\section{THREE-DIMENSIONAL} REPRESENTATION OF STRAIN TENSORS FOR A SHELL

The formulation (1.29) allows one to obtain the symmetrized tensor, i.e. the strain field: 


$$
\begin{aligned}
& \mathbf{e}=\boldsymbol{e}_{\alpha \beta} \mathbf{r}^{\alpha} \mathbf{r}^{\beta}+\boldsymbol{e}_{\alpha 3}\left(\mathbf{r}^{\alpha} \mathbf{n}+\mathbf{n} \mathbf{r}^{\alpha}\right)+e_{33} \mathbf{n n}= \\
& =\hat{e}_{\alpha \beta} \mathbf{R}^{\alpha} \mathbf{R}^{\beta}+\hat{e}_{\alpha 3}\left(\mathbf{R}^{\alpha} \mathbf{n}+\mathbf{n} \mathbf{R}^{\alpha}\right)+\hat{e}_{33} \mathbf{n n} ;
\end{aligned}
$$

the first formulation (1.32) is referred to the basis $\mathbf{r}^{\alpha}$ in $T S$ that depends not on $\zeta$, and

$$
\begin{aligned}
\breve{e}_{\alpha \beta}= & \frac{1}{2}\left(\nabla_{\beta} u_{\alpha}+\nabla_{\alpha} u_{\beta}\right)-\left(b_{\alpha \beta}+\zeta h K a_{\alpha \beta}\right) u_{3}- \\
& -\frac{1}{2} \zeta \mathrm{T}^{\gamma \lambda} b_{\lambda \cdot}^{\cdot \mu}\left(\mathrm{T}_{\alpha \mu} \nabla_{\gamma} u_{\beta}+\mathrm{T}_{\beta \mu} \nabla_{\gamma} u_{\alpha}\right) ; \\
\breve{e}_{\alpha 3}= & \frac{1}{2}\left(\nabla_{\alpha} u_{3}+b_{\alpha \cdot}^{\beta} u_{\beta}+\partial_{\zeta} u_{\alpha}\right)+\frac{1}{2} \zeta^{2} h^{2} K \partial_{\zeta^{\prime}} u_{\alpha}-( \\
- & \frac{1}{2} \zeta h\left(\mathrm{~T}^{\gamma \lambda} b_{\lambda \cdot \mathrm{T}_{\alpha \mu}}^{\cdot \mu} \nabla_{\gamma} u_{3}+K u_{\alpha}+2 H \partial_{\zeta} u_{\alpha}\right) ; \\
\breve{e}_{33}= & \left(1-2 \zeta h H+\zeta^{2} h^{2} K\right) \partial_{\zeta} u_{3} ; e_{i j}=A^{-1} \breve{e}_{i j} ;
\end{aligned}
$$

at the same time the second one (1.33) is referred to the main basis $\mathbf{R}^{\alpha}$ in $T S_{\zeta}$ :

$$
\begin{gathered}
\hat{e}_{\alpha \beta}=\frac{1}{2}\left(\nabla_{\alpha} u_{\beta}+\nabla_{\beta} u_{\alpha}\right)-\left(b_{\alpha \beta}+\zeta h c_{\alpha \beta}\right) u_{3}- \\
-\zeta h\left(b_{\alpha}^{\cdot \gamma} \nabla_{\beta} u_{\gamma}+b_{\beta}^{\cdot \gamma} \nabla_{\alpha} u_{\beta}\right) ; \\
\hat{e}_{\alpha 3}=\frac{1}{2}\left(\nabla_{\alpha} u_{3}+b_{\alpha \cdot}^{\cdot \beta} u_{\beta}+\partial_{\zeta} u_{\alpha}\right)- \\
-\zeta h b_{\alpha}^{\cdot \beta} \partial_{\zeta} u_{\beta} ; \quad \hat{e}_{33}=\partial_{\zeta} u_{3} .
\end{gathered}
$$

Let us note that both strain formulations use the translation components $u_{\alpha}, u_{3}$ referred to the basis $\mathbf{r}^{\alpha}$, $\mathbf{n}$ on the fibration $T^{\mathrm{e}} S \oplus N S$. Let us consider hence the tensor $\mathbf{e}_{S}$ :

$$
\begin{gathered}
\mathbf{e}_{s}=\hat{\mathbf{A}}^{\mathrm{T}} \cdot \mathbf{e} \cdot \hat{\mathbf{A}}, \quad \hat{\mathbf{A}}=\mathbf{A}+\mathbf{n n} ; \\
\mathbf{e}_{s}=\hat{e}_{\alpha \beta} \mathbf{r}^{\alpha} \mathbf{r}^{\beta}+\hat{e}_{\alpha 3}\left(\mathbf{r}^{\alpha} \mathbf{n}+\mathbf{n} \mathbf{r}^{\alpha}\right)+\hat{e}_{33} \mathbf{n n} .
\end{gathered}
$$

Accounting for (1.36) and (1.37) we have the inverse formula, i. e. $\mathbf{e}=\left(\hat{\mathbf{A}}^{-1}\right)^{\mathrm{T}} \cdot \mathbf{e}_{S} \cdot \hat{\mathbf{A}}^{-1}$.

\section{DIMENSIONAL REDUCTION}

Let $\mathrm{H}[-1,1]$ be a Hilbert space, and let us introduce a base function system on $\mathrm{H}$ [15]:

$$
\begin{gathered}
\mathrm{H}=\left\{\mathrm{p}_{(k)}(\zeta)\right\}=\left\{\mathrm{p}^{(m)}(\zeta)\right\}, \\
\left(\mathrm{p}_{(k)}, \mathrm{p}^{(m)}\right)=\delta_{(k)}^{(m)}
\end{gathered}
$$

(1.39) denotes the appropriate scalar product, thus, $p_{(k)}, p^{(m)}$ is a biorthogonal base system. Let us introduce a subspace $\mathrm{H}_{N}=\left\{p_{(k)}\right\}_{k=0 \ldots N}$, so that $\mathrm{H}=\mathrm{H}_{N} \oplus \Delta_{N}$. Taking into account (1.24), (1.38), and (1.39), we obtain $[14,15]$

$$
\begin{gathered}
\mathbf{u}=\mathbf{u}^{(k)} \mathrm{p}_{(k)}(\zeta), \quad \mathbf{u}^{(k)}=u_{\alpha}^{(k)} \mathbf{r}^{\alpha}+u_{3}^{(k)} \mathbf{n}, \\
\mathbf{u}^{(k)}=\left(\mathbf{u}, \mathrm{p}^{(k)}\right), \quad k=0 \ldots N
\end{gathered}
$$

The formula (1.40) approximates the spatial translation within $N+1$ Fourier coefficient

$$
\mathbf{u}^{(k)}: S \times(\square+\cup\{0\}) \rightarrow T^{\mathrm{c}} S \oplus N S
$$

being the vector fields on the Whitney sums of the cotangent and normal fibration of $S$.

Let us consider hence the strain tensor e defined by (1.34). Since this tensor is referred to $\mathbf{r}^{\alpha}, \mathbf{n}$, we could define them within a set of Fourier coefficients corresponding to (1.38):

$$
\mathbf{e}=\mathbf{e}^{(k)} \mathrm{p}_{(k)}(\zeta), \mathbf{e}^{(k)}=\left(\mathbf{e}, \mathrm{p}_{(k)}\right) .
$$

The Fourier coefficients $e^{(k)}$ define tensor fields on the fibration of the manifold $S$ :

$$
\begin{gathered}
\mathbf{e}^{(k)}: S \times\left(\square_{+} \cup\{0\}\right) \rightarrow \\
\rightarrow\left(T^{\mathrm{e}} S \oplus N S\right) \otimes\left(T^{\mathrm{e}} S \oplus N S\right) .
\end{gathered}
$$

On the other hand, let us consider a tensor

$$
\mathbf{e}_{A}=A \mathbf{e}, \quad A=\sqrt{g / a} ;
$$

defined by $\breve{e}_{\alpha \beta}, \breve{e}_{\alpha 3}, \breve{e}_{33}$; this tensor allows to represent its Fourier coefficients as follows: 


$$
\begin{gathered}
\breve{e}_{\alpha \beta}=\frac{1}{2}\left(\nabla_{\beta} u_{\alpha}^{(k)}+\nabla_{\alpha} u_{\beta}^{(k)}\right)- \\
-\left(b_{\alpha \beta} \delta_{(m)}^{(k)}+h K a_{\alpha \beta} Z_{(m)}^{(k)}\right) u_{3}^{(k)}- \\
-\frac{1}{2} Z_{(m)}^{(k)} \mathrm{T}^{\gamma \lambda} b_{\lambda \cdot}^{\mu}\left(\mathrm{T}_{\alpha \mu} \nabla_{\gamma} u_{\beta}^{(m)}+\mathrm{T}_{\beta \mu} \nabla_{\gamma} u_{\alpha}^{(m)}\right) ; \\
\breve{e}_{\alpha 3}^{(k)}=\frac{1}{2}\left(\nabla_{\alpha} u_{3}^{(k)}+b_{\alpha \cdot}^{\cdot \beta} u_{\beta}^{(k)}+D_{(n \cdot)}^{(\cdot m)} u_{\alpha}^{(n)}\right)- \\
-\frac{h}{2} Z_{(m)}^{(k)}\left(\mathrm{T}^{\gamma \lambda} b_{\lambda \cdot}^{\cdot \mu} \mathrm{T}_{\alpha \mu} \nabla_{\gamma} u_{\beta}^{(m)}+\right. \\
\left.+K u_{\alpha}^{(m)}+2 H D_{(n \cdot)}^{(\cdot m)} u_{\alpha}^{(n)}\right)+ \\
+\frac{1}{2} Z_{(l)}^{(k)} Z_{(m)}^{(l)} h^{2} K D_{(n \cdot)}^{(\cdot m)} u_{\alpha}^{(n)} ; \\
\breve{e}_{33}^{(k)}=\left(\delta_{(m)}^{(k)}-2 Z_{(m)}^{(k)} h H+\right. \\
\left.+Z_{(l)}^{(k)} Z_{(m)}^{(l)} h^{2} K\right) D_{(n \cdot)}^{(\cdot m)} u_{3}^{(n)} .
\end{gathered}
$$

Accounting for (1.32), (1.34), (1.43)-(1.44), we obtain the Fourier coefficients for $\mathbf{e}_{A}$ :

$$
\mathbf{e}_{A}=\mathbf{e}_{A}^{(k)} \mathrm{p}_{(k)}, \quad \mathbf{e}_{A}^{(k)}=\left(\mathbf{e}_{A}, \mathrm{p}^{(k)}\right) .
$$

The tensor e referred to the basis $\mathbf{R}^{\alpha}, \mathbf{n}$ and defined by (1.35) cannot be represented by surface tensors being its Fourier coefficients, but we could define the "shifted" tensor $\mathbf{e}_{S}$ :

$$
\mathbf{e}_{S}=\mathbf{e}_{S}^{(k)} \mathbf{p}_{(k)}, \quad \mathbf{e}_{S}^{(k)}=\left(\mathbf{e}_{S}, \mathbf{p}^{(k)}\right)
$$

the surface tensors $\mathbf{e}_{S}^{(k)}$ are determined by

$$
\begin{gathered}
\mathbf{e}_{S}^{(k)}=\hat{e}_{\alpha \beta}^{(k)} \mathbf{r}^{\alpha} \mathbf{r}^{\beta}+\hat{e}_{\alpha 3}^{(k)}\left(\mathbf{r}^{\alpha} \mathbf{n}+\mathbf{n} \mathbf{r}^{\alpha}\right)+\hat{e}_{33}^{(k)} \mathbf{n n} ; \\
\hat{e}_{\alpha \beta}^{(k)}=\frac{1}{2}\left(\nabla_{\alpha} u_{\beta}^{(k)}+\nabla_{\beta} u_{\alpha}^{(k)}\right)- \\
-\left(b_{\alpha \beta} \delta_{(m)}^{(k)}+h c_{\alpha \beta} Z_{(m)}^{(k)}\right) u_{3}^{(m)}- \\
-Z_{(m)}^{(k)} h\left(b_{\alpha \cdot}^{\cdot \gamma} \nabla_{\beta} u_{\gamma}^{(m)}+b_{\beta}^{\cdot \gamma} \nabla_{\alpha} u_{\alpha}^{(m)}\right) ; \\
\hat{e}_{\alpha 3}^{(k)}=\frac{1}{2}\left(\nabla_{\alpha} u_{3}^{(k)}+b_{\alpha \cdot}^{\cdot \beta} u_{\beta}^{(k)}+D_{(m \cdot)}^{(\cdot k)} u_{\alpha}^{(m)}\right)- \\
-h b_{\alpha \cdot}^{\cdot \beta} Z_{(m)}^{(k)} D_{(n \cdot)}^{(-m)} u_{\beta}^{(n)} ; \quad \hat{e}_{33}^{(k)}=D_{(n \cdot)}^{(\cdot m)} u_{3}^{(m)} .
\end{gathered}
$$

\section{STRESS TENSORS FOR A SHELL}

Let us introduce the following stress tensors:

$$
\begin{aligned}
& \boldsymbol{\sigma}=\sigma^{\alpha \beta} \mathbf{r}_{\alpha} \mathbf{r}_{\beta}+\sigma^{\alpha 3}\left(\mathbf{r}^{\alpha} \mathbf{n}+\mathbf{n} \mathbf{r}^{\alpha}\right)+\sigma^{33} \mathbf{n n}= \\
& =\hat{\sigma}^{\alpha \beta} \mathbf{R}_{\alpha} \mathbf{R}_{\beta}+\hat{\sigma}^{\alpha 3}\left(\mathbf{R}^{\alpha} \mathbf{n}+\mathbf{n} \mathbf{R}^{\alpha}\right)+\hat{\sigma}^{33} \mathbf{n n}
\end{aligned}
$$

this tensor defines the true stress as the Cauchy one in the nonlinear mechanics [18];

$$
\begin{aligned}
& \mathbf{s}=s^{\alpha \beta} \mathbf{r}_{\alpha} \mathbf{r}_{\beta}+s^{\alpha 3} \mathbf{r}_{\alpha} \mathbf{n}+s^{3 \beta} \mathbf{n r}_{\beta}+s^{33} \mathbf{n n}= \\
& =A\left[\hat{\sigma}^{\alpha \beta} \mathbf{r}_{\alpha} \mathbf{R}_{\beta}+\hat{\sigma}^{\alpha 3}\left(\mathbf{r}^{\alpha} \mathbf{n}+\mathbf{n} \mathbf{R}^{\alpha}\right)+\hat{\sigma}^{33} \mathbf{n n}\right] .
\end{aligned}
$$

Let us provide the analogy of the basis $\mathbf{r}^{\alpha}$ and the initial basis, as well as the basis $\mathbf{R}^{\alpha}$ and the acting basis in the nonlinear mechanics (i.e. the equidistant surface is obtained by motion of base surface points along normal). Thus, (1.50) is analogous to the Piola tensor;

$$
\boldsymbol{\pi}=A\left[\hat{\sigma}^{\alpha \beta} \mathbf{r}_{\alpha} \mathbf{r}_{\beta}+\hat{\sigma}^{\alpha 3}\left(\mathbf{r}^{\alpha} \mathbf{n}+\mathbf{n} \mathbf{r}^{\alpha}\right)+\hat{\sigma}^{33} \mathbf{n n}\right]
$$

is analogous to the $2^{\text {nd }}$ Piola stress tensor [18];

$$
\mathbf{s}=A \hat{\mathbf{A}}^{-1} \cdot \boldsymbol{\sigma}, \quad \boldsymbol{\pi}=A \hat{\mathbf{A}}^{-1} \cdot \boldsymbol{\sigma} \cdot\left(\hat{\mathbf{A}}^{-1}\right)^{\mathrm{T}} .
$$

Finally, let us introduce the tensor $\mathbf{t}=A \boldsymbol{\sigma}$ :

$$
\mathbf{t}=A\left[\sigma^{\alpha \beta} \mathbf{r}_{\alpha} \mathbf{r}_{\beta}+\sigma^{\alpha 3}\left(\mathbf{r}^{\alpha} \mathbf{n}+\mathbf{n} \mathbf{r}^{\alpha}\right)+\sigma^{33} \mathbf{n n}\right]
$$

analogous to the Trefftz-Kappus tensor [18].

Let us note that the tensors (1.48), (1.50), (1.52), (1.54) could be represented by their Fourier coefficients being surface tensors:

$$
\begin{array}{cc}
\boldsymbol{\sigma}=\boldsymbol{\sigma}_{(k)} \mathrm{p}^{(k)}, & \boldsymbol{\sigma}_{(k)}=\left(\boldsymbol{\sigma}, \mathrm{p}_{(k)}\right) ; \\
\mathbf{s}=\mathbf{s}_{(k)} \mathrm{p}^{(k)}, & \mathbf{s}_{(k)}=\left(\mathbf{s}, \mathrm{p}_{(k)}\right) ; \\
\boldsymbol{\pi}=\boldsymbol{\pi}_{(k)} \mathrm{p}^{(k)}, & \boldsymbol{\pi}_{(k)}=\left(\boldsymbol{\pi}, \mathrm{p}_{(k)}\right) ; \\
\mathbf{t}=\mathbf{t}_{(k)} \mathrm{p}^{(k)}, & \mathbf{t}_{(k)}=\left(\mathbf{t}, \mathrm{p}_{(k)}\right) .
\end{array}
$$


On Different Definitions of Strain Tensors in General Shell Theories of Vekua-Amosov Type

\section{STRAIN ENERGY FORMULATION}

Accordingly to [16], we have,

$$
U=\frac{1}{2} \int_{V} \boldsymbol{\sigma}: \mathbf{e} d V=\frac{1}{2} \int_{S}^{1} \int_{-1}^{1} \boldsymbol{\sigma}: \mathbf{e} A h d \zeta d S .
$$

Let us define a surface strain energy density:

$$
U_{S}=\int_{-1}^{1} W_{S} h d \zeta, \quad W_{S}=\frac{1}{2} A \boldsymbol{\sigma}: \mathbf{e}
$$

Here the strain energy density $W_{S}$ could be represented using the equivalent formulations, e.g. we have the work-conjugated tensor pairs

$$
2 W_{S}=\boldsymbol{\sigma}: \mathbf{e}_{A}=\mathbf{s}: \mathbf{D}=\boldsymbol{\pi}: \mathbf{e}_{S}=\mathbf{t}: \mathbf{e}
$$

where $\mathbf{D}$ is the reduced distortion tensor [15]:

$$
\begin{gathered}
\mathbf{D}=\left(\mathbf{A}^{-1}\right)^{\mathrm{T}} \cdot \mathbf{d}= \\
=\bar{d}_{\alpha \beta} \mathbf{r}^{\alpha} \mathbf{r}^{\beta}+\bar{d}_{\alpha 3} \mathbf{r}^{\alpha} \mathbf{n}+\bar{d}_{3 \beta} \mathbf{n} \mathbf{r}^{\beta}+\bar{d}_{33} \mathbf{n n} .
\end{gathered}
$$

Accounting for (1.42)-(1.47), (1.48)-(1.55) as well as for (1.38) and (1.39), we obtain

$$
\begin{aligned}
& 2 U_{s}=\sigma_{(k)}^{\alpha \beta} \breve{e}_{\alpha \beta}^{(k)}+2 \sigma_{(k)}^{\alpha 3} \breve{e}_{\alpha 3}^{(k)}+\sigma_{(k)}^{33} \breve{e}_{33}^{(k)}, \\
& \sigma_{(k)}^{\ddot{j}}=h\left(\sigma^{i j}, \mathrm{p}_{(k)}\right) ; \\
& 2 U_{S}=\pi_{(k)}^{\alpha \beta} \hat{e}_{\alpha \beta}^{(k)}+2 \pi_{(k)}^{\alpha 3} \hat{e}_{\alpha 3}^{(k)}+\pi_{(k)}^{33} \hat{e}_{33}^{(k)}, \\
& \pi_{(k)}^{\bar{y}}=h\left(A \hat{\sigma}^{j}, \mathrm{p}_{(k)}\right) ; \\
& 2 U_{S}=t_{(k)}^{\alpha \beta} e_{\alpha \beta}^{(k)}+2 t_{(k)}^{\alpha 3} e_{\alpha 3}^{(k)}+t_{(k)}^{33} e_{33}^{(k)}, \\
& t_{(k)}^{i j}=h\left(A \sigma^{i j}, \mathrm{p}_{(k)}\right) ; \\
& 2 U_{S}=s_{(k)}^{\alpha \beta} \bar{d}_{\alpha \beta}^{(k)}+s_{(k)}^{\alpha 3} \bar{d}_{\alpha 3}^{(k)}+s_{(k)}^{3 \beta} \bar{d}_{3 \beta}^{(k)}+s_{(k)}^{33} \bar{d}_{33}^{(k)}, \\
& s_{(k)}^{i j}=h\left(s^{i j}, \mathrm{p}_{(k)}\right) \text {. }
\end{aligned}
$$

The formula (1.63) was used to derive the dynamic equations in [14] and [15].

\section{STRAINS AND STRAIN ENERGY FOR THE FIRST-ORDER SHELL THEORY}

Let us consider hence the $1^{\text {st }}$ order shell theory as a particular case of the $\mathrm{N}^{\text {th }}$ order:

$$
\mathbf{u}=\mathbf{u}^{(0)}+\zeta \mathbf{u}^{(1)} .
$$

Here $\mathbf{u}^{(0)}=\bar{u}_{\alpha} \mathbf{r}^{\alpha}+w \mathbf{n}$ so that corresponds to (1.7); $\mathbf{u}^{(1)}=\chi_{\alpha} \mathbf{r}^{\alpha}+\psi \mathbf{n}$, here the displacement amplitude $\chi_{\alpha}$ corresponds to the tangent rotation angle, $\varphi^{\alpha}=\mathbf{T}^{\alpha \beta} \chi_{\beta}$, while $\psi$ is the transverse normal strain. Thus, we have

$$
\begin{gathered}
\nabla \otimes \mathbf{u}^{(0)}=\mathbf{d}, \quad \mathbf{D}=\mathbf{d}+\psi \mathbf{n} \mathbf{n}+\zeta \mathbf{d}^{(1)} ; \\
\mathbf{d}^{(1)}=d_{\alpha \beta}^{(1)} \mathbf{R}^{\alpha} \mathbf{r}^{\beta}+d_{\alpha 3}^{(1)} \mathbf{R}^{\alpha} \mathbf{n} . \\
d_{\alpha \beta}^{(1)}=\nabla_{\alpha} \chi_{\beta}-b_{\alpha \beta} \psi ; d_{\alpha 3}^{(1)}=\nabla_{\alpha} w+b_{\alpha \cdot}^{\beta} \cdot \bar{u}_{\beta} .
\end{gathered}
$$

Accordingly to (1.11), (1.13), (1.35), (1.65), we obtain the following $1^{\text {st }}$ order approximation of the strain tensor (1.37):

$$
\begin{gathered}
\hat{e}_{\alpha \beta}=\varepsilon_{\alpha \beta}+\zeta \amalg_{\alpha \beta}-\zeta b_{\alpha \beta} \psi- \\
-\frac{1}{2} \zeta^{2}\left(b_{\cdot \alpha}^{\gamma} \cdot d_{\beta \gamma}^{(1)}+b_{\beta}^{\cdot \gamma} \cdot d_{\alpha \gamma}^{(1)}\right) ; \\
\hat{e}_{\alpha 3}=\frac{1}{2}\left(\vartheta_{\alpha}+\chi_{\alpha}\right)+\frac{1}{2} \zeta \nabla_{\alpha} \psi, \quad \hat{e}_{33}=\psi .
\end{gathered}
$$

Thus, the reduced strain tensor (1.37) of the $1^{\text {st }}$ approximation referred to the basis $\mathbf{r}^{\alpha}, \mathbf{n}$ on the fibration $T S \otimes N S$ is given by the surface strains, i.e. tangent strain (1.11) and bending strain (1.13) of the surface as well as the averaged transverse shear $\theta_{\alpha}$, splitting shear $\nabla_{\alpha} \psi$ [7] and the normal transverse $\psi$.

Let us consider hence the true strain tensor $\mathbf{e}$ in terms of the $1^{\text {st }}$ order approximation; it is given by the covariant components (1.66), (1.67). Since this tensor is referred to the basis $\mathbf{R}^{\alpha}, \mathbf{n}$ on the fibration $T_{\zeta}^{\mathrm{e}} S \otimes N S$ it does not allow one to create a set of surface tensors by the integration over the thickness. 
Let us represent this tensor by the particular sum of Taylor series with respect to $\zeta$; since we have the formula for the base vectors $\mathbf{R}^{\alpha}$.

$$
\left.\mathbf{R}^{\alpha} \approx \mathbf{R}^{\alpha}\right|_{\zeta=0}+\left.\zeta \partial_{\zeta} \mathbf{R}^{\alpha}\right|_{\zeta=0}=\left(\delta_{\beta}^{\alpha}+\zeta b_{\beta}^{\cdot \alpha}\right) \mathbf{r}^{\beta},
$$

Use of the Taylor series together with (1.68) gives the following strain tensor $\mathbf{e}$ :

$$
\begin{gathered}
\mathbf{e}=e_{\alpha \beta} \mathbf{r}^{\alpha} \mathbf{r}^{\beta}+e_{\alpha 3}\left(\mathbf{r}^{\alpha} \mathbf{n}+\mathbf{n} \mathbf{r}^{\alpha}\right)+e_{33} \mathbf{n n}, \\
e_{\alpha \beta} \approx \varepsilon_{\alpha \beta}+\zeta \kappa_{\alpha \beta}, \\
\kappa_{\alpha \beta}=щ_{\alpha \beta}+b_{\cdot \alpha}^{\gamma} \varepsilon_{\beta \gamma}+b_{\cdot \beta}^{\gamma} \varepsilon_{\alpha \gamma} ;
\end{gathered}
$$

thus, the first-order term obtained by Taylor expansion gives the reduced bending strain tensor $\kappa_{\alpha \beta}$ corresponding to the one used in classical shell theories (e. g. see [2, 3]).

Let us note that the tangent strain $\varepsilon_{\alpha \beta}$ and the reduced bending strain $\kappa_{\alpha \beta}$ definig the tensor $\mathbf{e}_{\mathrm{T}}=$ $e_{\alpha \beta} \mathbf{r}^{\alpha} \mathbf{r}^{\beta}$ are two quantities being unknowns in the compatibility equations of the classical theory of thin shells [2]:

$$
\begin{aligned}
& \nabla_{\beta} \mathrm{T}^{\beta \gamma} \mathrm{T}^{\alpha \delta} \kappa_{\gamma \delta}-b_{\beta}^{\alpha} \nabla_{\delta} \mathrm{T}^{\beta \gamma} \mathrm{T}^{\alpha \delta} \varepsilon_{\gamma \delta}=0 ; \\
& \nabla_{\alpha} \nabla_{\beta} \mathrm{T}^{\alpha \gamma} \mathrm{T}^{\beta \delta} \varepsilon_{\gamma \delta}+b_{\alpha \beta} \mathbf{T}^{\alpha \gamma} \mathrm{T}^{\beta \delta} \kappa_{\gamma \delta}=0,
\end{aligned}
$$

that provide the static-geometry analogy [2]. Accounting for (1.62), (1.33) and introducing averaged shear $\theta_{\alpha}=\vartheta_{\alpha}+\chi_{\alpha}$, we have hence

$$
\begin{gathered}
2 U_{S}=\pi_{(0)}^{\alpha \beta} \varepsilon_{\alpha \beta}+2 \pi_{(0)}^{\alpha 3} \theta_{\alpha}+\pi_{(0)}^{33} \psi+ \\
+\pi_{(1)}^{\alpha \beta}\left(\Psi_{\alpha \beta}^{(1)}-b_{\alpha \beta} \psi\right)+2 \pi_{(1)}^{\alpha 3} \nabla_{\alpha} \psi, \\
\pi_{(0)}^{\alpha \beta}=h \int_{-1}^{1} \hat{\sigma}^{\alpha \beta} A d \zeta, \pi_{(1)}^{\alpha \beta}=h \int_{-1}^{1} \hat{\sigma}^{\alpha \beta} A \zeta d \zeta, \\
\pi_{(0)}^{\alpha 3}=h \int_{-1}^{1} \hat{\sigma}^{\alpha 3} A d \zeta, \pi_{(1)}^{\alpha 3}=h \int_{-1}^{1} \hat{\sigma}^{\alpha 3} A \zeta d \zeta ; \\
\pi_{(2)}^{\alpha \beta}=\frac{h}{2} \int_{-1}^{1} \hat{\sigma}^{\alpha \beta} A \zeta^{2} d \zeta ; \pi_{(1)}^{33}=\int_{-1}^{1} \hat{\sigma}^{33} A d \zeta .
\end{gathered}
$$

Here $\pi_{(0,1)}^{\alpha \beta}, \pi_{(0,1)}^{\alpha 3}, \pi_{(0)}^{33}$ are expansion factors of the " 2 nd Piola" tensor $\pi$, or the moments of the contravariant components of the true strain tensor $\hat{\sigma}^{i j}$. Here the tensor given by $\pi_{(0)}^{\alpha \beta}$ works on the tangent strain $\varepsilon_{\alpha \beta}$ (1.11), the tensor given by $\pi_{(1)}^{\alpha \beta}$ works on the bending strain $щ_{\alpha \beta}$ (1.13) (and $b_{\alpha \beta} \psi$ ), while $\pi_{(0)}^{\alpha 3}$ works on the averaged shear $\theta_{\alpha}$. Thus, $\pi_{(0)}^{\alpha \beta}$ defines the work-conjugated tangent force tensor, $\pi_{(1)}^{\alpha \beta}$ defines the work-averaged bending couple, while $\pi_{(0)}^{\alpha 3}$ is the workaveraged shear force and finally $\pi_{(1)}^{\alpha 3}$ is the work-averaged splitting force [7]. Let us note that these quantities differ from the ones working on $\delta \bar{u}_{\alpha}, \delta \chi_{\alpha}$ and being unknown quantities in the dynamic equations [15]; the last ones were defined as $\mathbf{s}_{(0)}, \mathbf{s}_{(1)}, \ldots$ being surface tensors on $T S \oplus N S$ [7]. Accounting for the formulae for the contravariant components of the stress tensor $\mathbf{s}$ [15]:

$$
\begin{gathered}
s^{\alpha \beta}=A A_{\cdot \gamma}^{\beta \cdot} \sigma^{\alpha \gamma}=A A_{\delta \cdot}^{\alpha \alpha} \hat{\sigma}^{\delta \beta}, \\
s^{3 \beta}=A A_{\cdot \gamma}^{\beta \cdot} \sigma^{3 \gamma}=A \hat{\sigma}^{3 \beta}, s^{\alpha 3}=A \sigma^{\alpha 3}=A A_{\delta \cdot}^{\alpha} \hat{\sigma}^{\delta 3},
\end{gathered}
$$

we obtain the quantities equivalent to the ones introduced in [19]. The stresses used in classical shell theories [2] appears as a result of neglecting of the value $\mathrm{A}=\sqrt{g / a}$ (e.g. see [2]), the similar assumption was used by I.N.Vekua for the general theory of thin shallow shells [7]; such a tensor could be obtained as $\mathbf{S}=\mathbf{A}^{-1} \cdot \boldsymbol{\sigma}$, therefore we have

$$
S_{(0)}^{\alpha \beta}=h \int_{-1}^{1} A_{\gamma \cdot}^{\alpha} \hat{\sigma}^{\gamma \beta} d \zeta, S_{(1)}^{\alpha \beta}=h \int_{-1}^{1} A_{\gamma}^{\alpha \alpha} \hat{\sigma}^{\gamma \beta} \zeta d \zeta .
$$

On the other hand one could consider the strain energy (1.62) with the components of the strain $e_{i j}^{(k)}$ defined by the formula (1.69); this is the formulation that uses the tangent and bending strains accordingly to [2]. Thus, we obtain the 
following definitions of the tangent force and bending moment:

$$
t_{(0)}^{\alpha \beta}=h \int_{-1}^{1} t^{\alpha \beta} d \zeta, \quad t_{(1)}^{\alpha \beta}=h \int_{-1}^{1} t^{\alpha \beta} \zeta d \zeta ;
$$

here the contravariant components $t^{i j}=A \sigma^{i j}$ of the stress tensor referred to the basis $\mathbf{r}_{\alpha}, \mathbf{n}$ in the fibration $T S \otimes N S$ are used contrarily to where the ones referred to the basis $\mathbf{R}^{\alpha}, \mathbf{n}$ in the fibration $T_{\zeta} S \otimes N S$ were used. The definition of tangent forces and couples tensors corresponding to [2] could be obtained by neglecting the multiplier $A$.

\section{CONCLUSIONS}

One can conclude that several different strain tensors could be considered. These definitions are required, in particular, to derive the constraint equations for extended higher-order shell theories [20] that appears a result of the translation of boundary conditions from faces onto a base surface. The further construction of extended shell theories without the use of constraint multiplier could be based on the constraint equations represented in terms of generalized strains. Moreover, the statement of the boundary value problem of the higher-order shell theory in terms of generalized forces requires the appropriate compatibility equations such as the ones proposed in [22] where only the 1st order shell model was considered; various definitions of strain tensors generate different formulations of the two-dimensional compatibility equations. On the other hand the solution of problems of coupled dynamics of medium-thickness shells and surrounding media (e.g. see [23, 24]) require constraint equations corresponding to the impermeability condition on wetted shell face; such equation could be also expressed in terms of strains, thus, an approach similar to [2] where the strain boundary conditions were defined on shells' contours. Finally, the solution of different static problems $[25,26]$ could be more efficient if the stress-based problem statement is used as a background.

\section{ACKNOWLEDGEMENTS}

This investigation was performed as a part of the State Task for Basic Researches of Institute of Applied Mechanics of Russian Academy of Sciences and partially supported also by the RFBR under the grants Nr. 19-01-00695-a and Nr. 2008-0891-a.

\section{REFERENCES}

1. Kilchevskii N.A. Basics of the analytical mechanics of shells (in Russian). Kiev, Izd. Akademy of Sci. of USSR, 1963.

2. Novozhilov V.V., Chernykh K.F., Mikhailovskii E.I. Linear theory of thon shells. Leningrad, Politekhnika, 1991.

3. Goldenveizer A.L. Theory of thin elastic shells. Moscow, Fizmatlit, 1976.

4. Eremeyev V.A., Zubov L.M. Mechanics of elastic shells. Moscow, Nauka, 2008.

5. Pelekh B.L. Theory of shells with finite shear stiffness. Kiev, Nauk. Dumka, 1973.

6. Pelekh B.L. Generalized theory of shells. Kiev, Vishcha Shkola, 1978.

7. Vekua I.N. Shell Theories: General Methods of Construction. Boston, USA: Pitman Adv. Publ. Progr., 1985.

8. Yokoo Y., Matsunaga H. A general nonlinear theory of elastic shells // Int. J. Sol. Struct. 1974, vol. 10, pp. 261-274.

9. Guliaev V.I., Bazhenov B.A., Lizunov P.P. Nonclassical Shell Theory and Applications in Engineering (in Russian), Lvov, Vishcha Shkola, 1978.

10. Khoma I.Yu. Generalized theory of anisotropic shells. Kiev: Naukova Dumka, 1986.

11. Amosov A.A. An approximate threedimensional theory of thick plates and shells // Structural Mechanics and Design of Buildings, No. 5 (1987), pp. 37-42.

12. Amosov A.A. An approximate threedimensional theory of non-thin elastic shells: Diss. Dr. Sci. Tashkent State University, Tashkent, 1989. 
13. Nikabadze M.U. Method of orthogonal polynomials in mechanics of micropolar and classical elastic solids. Dr. Sci. Diss. Moscow State Univ. 2014.

14. Zhavoronok S.I. Variational equations of a three-dimensional anisotropic theory of shells // Vestn. Lobachevsky Univ. N. Novgorod, Iss. 4-5 (2011). Pp. 2154-2156.

15. Zhavoronok S.I. A Vekua-type linear theory of thick elastic shells // ZAMM - Zeitschrift für Angewandte Mathematik und Mechanik. Vol. 92, No. 1-2 (2014). Pp. 164-184.

16. Abovskii N.P., Andreev N.P., Deruga A.P. Variational principles of the elasticity theory and shell theory (in Russian), Moscow, Fizmatlit, 1978.

17. Amosov A.A., Zhavoronok S.I. An approximate high-order theory of thick anisotropic shells // International Journal of Computational Civil and Structural Engineering. Vol. 1 (2003), pp. 28-38.

18. Lurie A.I. Nonlinear theory of elasticity. North Holland, 1990.

19. Pobedrya B.E., Georgievskiy D.V. Lectures on Elasticity Theory. Moscow, Editorial URSS, 1999.

20. Zhavoronok S.I. The generalized Lagrange equations of the second kind for the extended three-dimensional n'th order theory of anisotropic shells // The J. of Mekhanika Kompozitsionnykh Materialov I Konstruktsii. Vol. 21 (2015), pp. 370-381.

21. Zhavoronok S.I. The extended shell theory of Vekua-Amosov type and the low- order plate models // International Journal for Computational Civil and Structural Engineering. Vol. 12 (2016), pp. 27-35.

22. Tarlakovskii D.V., Zhavoronok S.I. On the compatibility equations in shell theories considering transverse shear and normal strains. In: Shell Structures: Theory and Applications, Vol. 4. CRC Press / Balkema, Taylor \& Francis Gr., Leiden (2018), pp. 189-192.

23. Rabinsky L.N., Zhavoronok S.I. Axisymmetric problem of unsteady interaction of acoustic pressure waves with elastic shell of revolution // The J. of Mekhanika Kompozitsionnykh Materialov I Konztruktsii. Vol.12 (2006), pp. 541-554.

24. Egorova O.V., Rabinskiy L.N., Zhavoronok S.I. Middle thickness shell's interaction with acoustical wave // Aerospace MAI Journal. Vol.17 (2010), pp. 127-135.

25. Zhavoronok S.I., Leontiev A.N., Leontiev K.A. Analysis of thick-walled rotation shells based on the Legendre polynomials // International Journal for Computational Civil and Structural Engineering. Vol. 6(2010). Pp. 105-111.

26. Amosov A.A., Zhavoronok S.I., Leontiev K.A. About solving some problem on the stressed-strained state thick anisotropic shells of revolution in three-dimensional statement // The J. of Mekhanika Kompozitsionnykh Materialov I Konstruktsii. Vol.10 (2010), pp. 301-310.
Zhavoronok Sergey Igorevich, Ph.D., Senior researcher of the Department of Mechanics of Smart and Composite Materials and Systems, Institute of Applied Mechanics of Russian Academy of Sciences (IAM RAS); Associate Professor of the Department of Strength of Materials,
Machine Dynamics and Strength, Moscow Aviation Institute (National Research University) - MAI. Address: Leningradskiy Prospekt 7, 125040, Moscow, Russia, IAM RAS. Phone: +7(495) 941-1777; +7(916) 134-2843. e-mail: Zhavoronok@iam.ras.ru, zhavor71@mail.ru 